VIII ${ }^{\text {èmes }}$ Journées Nationales Génie Civil - Génie Côtier, Compiègne, 7-9 septembre 2004

\title{
Les problèmes hydrauliques de calcul d'ouvrages de prise d'eau et de rejet en mer
}

\author{
M.Hassane(a), M.K. Mihoubi(b), M.S.Benhafid(c) \\ École Nationale Supérieure de l'Hydraulique de Blida, BP 31Blida (09000)-ALGERIE \\ Tel: $+213.25 .39 .94 .47 ;$ Fax: +213.25 .39 .94 .46$ \\ E-mail:(a) hassanemed@yahoo.fr; (b) mihkam@yahoo.fr; (c) mohbenhafid@yahoo.fr
}

\section{Résumé:}

Contrairement aux ouvrages de même type projetés sur des rivières ou sur des bassin de retenue, les ouvrages de prise et de rejet d'eau en mer, destinés au refroidissement des turbines de centrales thermiques et nucléaires, subissent les effets de l'interaction de la houle et des courants dans la zone de leur influence. L'implantation de tels ouvrages de moyenne et grande importance nécessite une étude préalable complète et un calcul hydraulique qui tient compte de la transformation des paramètres hydrodynamiques de la houle par les courants. Cette transformation a pour conséquence la modification des conditions de déferlement et de blocage des vagues qui influent considérablement sur la stabilité de l'ouvrage et sur l'évolution et la configuration du trait de côte du rivage contigu. Dans la présente communication nous proposons un schéma général de calcul hydraulique des ouvrages de prise et de rejet d'eau en mer. L'étude de l'action combinée de la houle et des courants nous a permis de définir les paramètres hydrodynamiques à prendre en considération dans les calculs de stabilité des ouvrages et du transport des sédiments dans la zone d’influence.

\section{Introduction}

Les projets de construction des centrales thermiques et nucléaires posent des problèmes naturels de calcul de prises et de rejet d'eau, de capacités considérables, nécessaires au refroidissement des turbines. Pour les ouvrages de prise d'eau ou de rejet projetés sur un grand cours d'eau ou sur un bassin de retenue, où le mouvement des vagues est insignifiant, le problème se rapporterait à des questions suffisamment étudiées de conception et d'argumentation hydraulique de l'ouvrage (calcul de capacité de débit, de l'ensablement, de l'envahissement des mauvaises herbes, des conditions de stratification etc....). Ces questions sont bien élucidées dans les travaux [1,4]. Il existe d'autres travaux qui, dans la conception des ouvrages de prise d'eau ou de rejet, prennent en considération l'influence des vagues, des courants littoraux, de l'ensablement dû à l'effet des vagues, de l'agitation etc....[2,3,4]

Cependant dans le cas de prise d'eau ou de rejet en mer, les effets de la houle jouent un rôle essentiel. Il est donc indispensable d'effectuer une recherche de méthodes hydrauliques de calcul, tant du canal d'amenée que des éléments de son raccordement aval. Ces méthodes doivent prendre en considération: les courants littoraux dans la zone d'influence de l'ouvrage, le transport des sédiments par charriage et par suspension, les paramètres de la houle et la tendance générale de la dynamique des bandes côtières. 


\section{Problématique}

Par la figure $\mathrm{N}^{\circ} 01$ nous avons schématisé le calcul des prises d'eau ou de rejet en mer. Dans la présente communication, une attention particulière a été accordée à la question de prise en compte des courants, de la houle et de leur interaction, dans le calcul des ouvrages de rejet en mer. L'influence de la houle sur les conditions de fonctionnement de l'ouvrage de prise d'eau ou de rejet est liée aux effets suivants :

D'abord, les paramètres des vagues agissantes directement sur l'ouvrage la tête de l'ouvrage sont influencés non seulement par les conditions hydrométéorologiques et la topographie du fond, mais aussi par les courants dans la zone d'influence de l'ouvrage. Ces courants sont des courants superficiels, littoraux, de marrée et autres. Les courants de rencontre peuvent entraîner des accroissements de l'amplitude de la vague de deux fois et plus. Dans ce cas, des effets de déferlement et de blocage de la vague peuvent être observés. Dans la zone de déferlement de la vague sur le courant de rencontre, les vitesses moyennes de fond et l'intensité de turbulence augmentent brusquement, ce qui entraîne des affouillements (local et général) considérables dans la zone de raccordement. Il est donc indispensable de prendre en considération ces effets dans les calculs de stabilité des ouvrages de raccordement et dans la précision des profondeurs d'affouillement local à l'aval de l'ouvrage. Dans le cas d'une incidence oblique de la houle par rapport à la direction du courant, on observe de plus, l'effet de réflexion de la houle sur les frontières de la veine liquide. Par ailleurs les lames debout peuvent augmenter l'angle d'étalement et diminuer les grandeurs des vitesses longitudinales du jet comparativement à l'étalement du jet en l'absence de la houle.

Les courants de marrée peuvent sensiblement changer les conditions de génération de la houle de vent et par conséquent les conditions de fonctionnement des ouvrages et la dynamique des sédiments sur le littoral. De tels problèmes sont importants pour les grands canaux et estuaires.

Les courants littoraux déterminent le régime sédimentaire dans la zone de rivage. Les matériaux transportés par les courants littoraux peuvent se déposer à l'aval de l'ouvrage, altérant ainsi les conditions de fonctionnement de prise d'eau et de rejet. Cependant une dérivation provoquée par la configuration du fond de type barre marine est fort possible, ce qui peut influer sur la disposition générale des ouvrages. Dans le but d'une optimisation des ouvrages de prise d'eau ou de rejet et de résolution des problèmes de garantie de fonctionnement de l'aménagement entier des ouvrages, et de prévention des effets négatifs de déformation du rivage dans la zone d'influence de l'ouvrage ; il est indispensable d'effectuer des calculs complexes d'interaction de la houle et des courants. Les résultats expérimentaux ainsi que les bases de calcul pour un certain nombre de problèmes hydrauliques de l'action combinée de la houle et des courants sont données ci-dessous. 


\section{Ouvrage maritime de prise d'eau /de rejet}

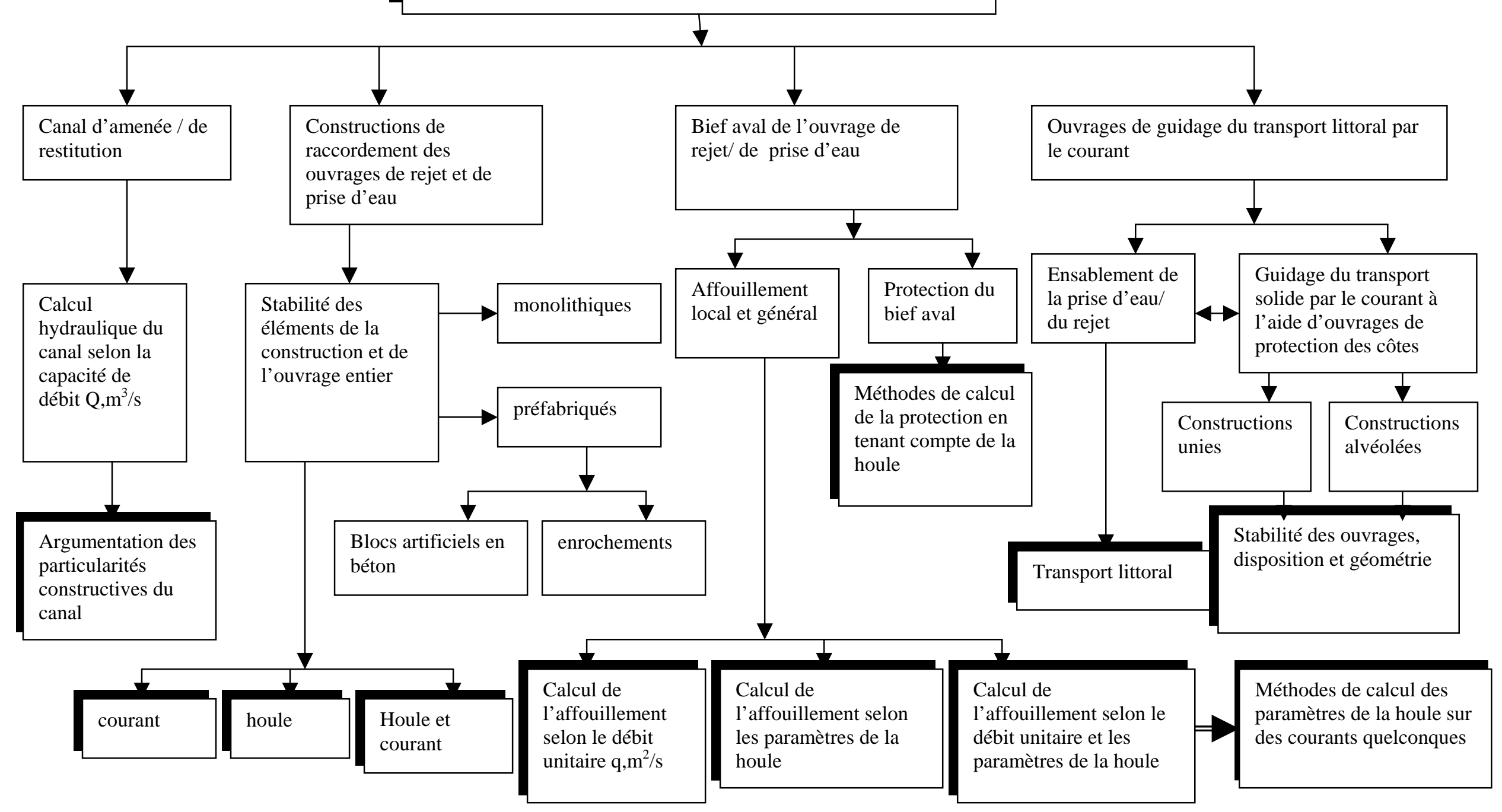

Figure 1: Schéma de calcul des ouvrages maritimes de rejet et de prise d'eau 


\section{3.Étude de l’action combinée de la houle et des courants:}

Un grand ensemble de problèmes de l'action combinée de la houle et des courants du point de vue hydrotechnique sont examinés dans les travaux [5,6,7,20,21].

Dans le cas de génération d'ondes de surface, due à l'action du vent sur un plan d'eau illimité et en l'absence de courant, il a été vérifié expérimentalement qu'il existe des dépendances entre les grandeurs adimensionnelles de l'amplitude et de la période de la houle d'une part et les grandeurs adimensionnelles de la longueur de fetch et de la profondeur d'autre part [ 8,9]:

$\frac{g \mathrm{H}}{\mathrm{W}^{2}}, \frac{\mathrm{gT}}{\mathrm{W}}\left(\frac{\mathrm{gx}}{\mathrm{W}^{2}}, \frac{\mathrm{gd}}{\mathrm{W}^{2}}\right)$

où: H- amplitude de la houle (moyenne ou la plus grande) ; W- vitesse théorique du vent ; $\mathrm{T}$ - période de la houle (moyenne ou correspondante à la fréquence du maximum du spectre) ; x- longueur du fetch ; d- profondeur ; g- accélération de la pesanteur.

Pour la génération d'ondes sur les courants de marrée apparaît un nouveau facteur, que l'on peut présenter comme vitesse adimensionnelle du courant: $\frac{U}{W}$. Ce facteur influe sur la longueur effective du fetch, qui se détermine par le rapport du temps de l'interaction du vent et de la surface agitée pour l'action combinée de la houle et du courant sur le temps de cette interaction en l'absence du courant :

$$
\frac{X_{\text {eff }}}{X}=\frac{\int_{0}^{x} \frac{d x}{\overline{\bar{C}_{g a}(x)}}}{\int_{0}^{x} \frac{d x}{\overline{c_{g 0}(x)}}}
$$

où: $\overline{\mathrm{C}_{\mathrm{ga}_{\mathrm{a}}}}, \overline{\mathrm{C}_{\mathrm{g} 0}}$ - vitesses moyennes absolues de groupe, respectivement en eau courante et en eau statique.

Le calcul de $\frac{X_{\text {eff }}}{\mathrm{X}}$ en fonction de $\frac{U}{W}$ et $\frac{\mathrm{gx}}{\mathrm{W}^{2}}$ est représenté par le graphique de la figure $\mathrm{N}^{\circ} 02$. Pour le courant de rencontre $\left.\left(\frac{X_{\text {eff }}}{X}\right) 1\right)$, l'amplitude et la longueur d'onde sont plus grandes qu'en eau statique, par contre pour le courant favorable $\left(\frac{x_{\text {eff }}}{x}\langle 1)\right.$,les ondes sont plus petites qu'en l'absence de courant.

L'application de la relation (2) est basée sur l'analyse du modèle de l'équation de la variation de la densité de l'action ondulatoire, qui pour la propagation de la houle sur un courant stationnaire graduellement varié, est de la forme :

$$
\frac{\partial \mathrm{N}}{\partial \mathrm{x}} \cdot \mathrm{C}_{\mathrm{ga}}=\mathrm{S}
$$

où : $\mathrm{N}(\mathrm{k}, \mathrm{x})$ - densité spectrale de l'action ondulatoire, $\mathrm{C}_{\mathrm{ga}^{-}}$vitesse absolue de groupe d'ondes harmoniques $\mathrm{k}, \mathrm{S}$ - fonction de source, déterminant le transfert d'énergie des ondes dues à l'action du vent à la surface agitée, la dissipation et les interactions non linéaires. 


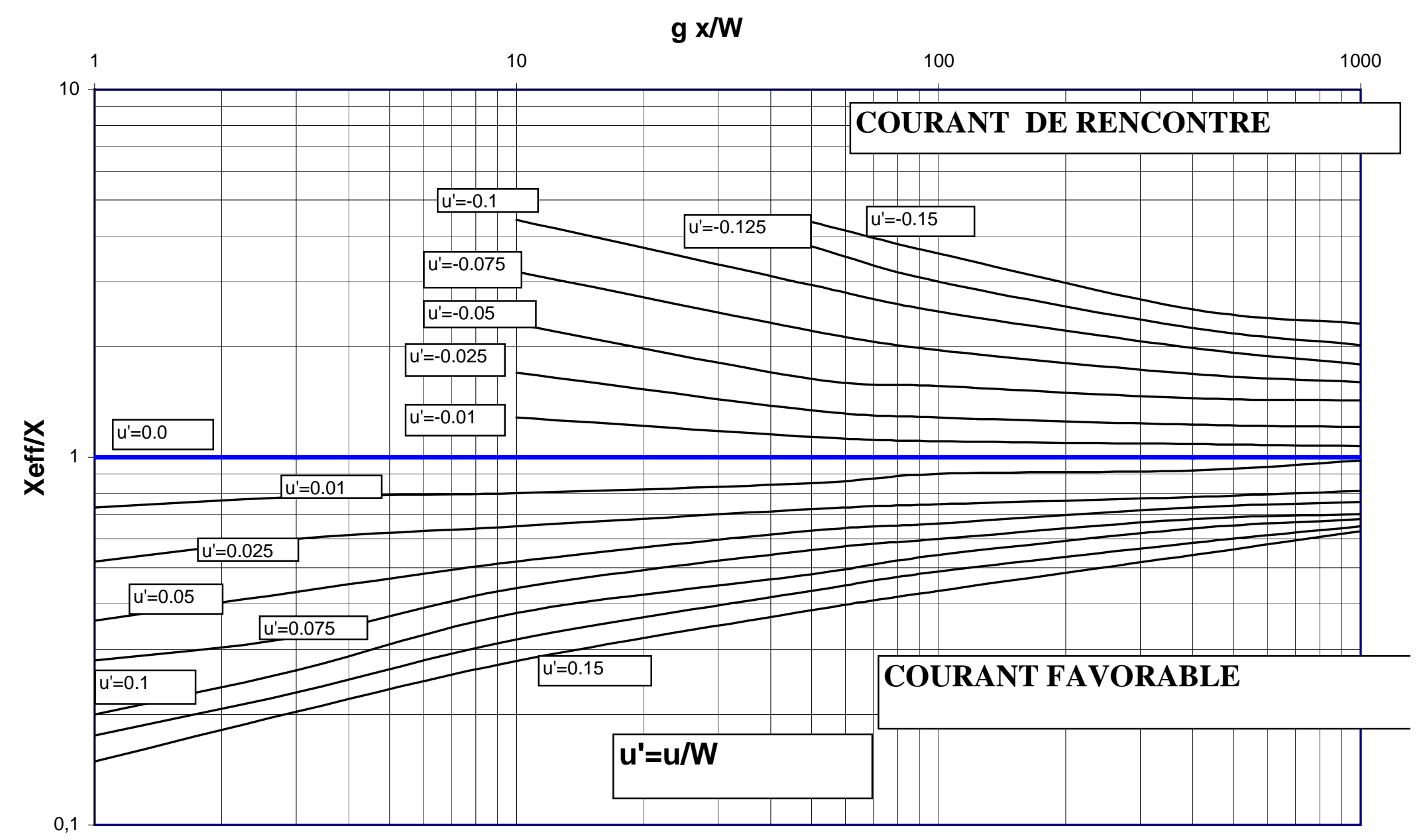

Figure 2 : Graphique de détermination de xeff/x 
Si pour chaque composante spectrale, nous effectuons, dans la relation (3), la transformation suivante:

$$
\frac{\mathrm{X}}{\mathrm{X}}=\frac{\mathrm{C}_{\mathrm{ga}}}{\mathrm{C}_{\mathrm{g} 0}}
$$

Nous obtenons alors:

$\frac{\partial \mathrm{N}}{\partial \mathrm{x}} \cdot \mathrm{C}_{\mathrm{g} 0}=\mathrm{S}^{\prime}$

où $S^{\prime}$ - fonction de source obtenue après transformation correspondante de $S$. Si l'on posait $S^{\prime}=S_{0}$ (où : $S_{0}-$ fonction de source en l'absence de courant), ainsi la relation (5) coïncide avec l'équation de variation de la densité de l'action ondulatoire en l'absence de courant:

$\frac{\partial \mathrm{N}_{0}}{\partial \mathrm{x}} \cdot \mathrm{C}_{\mathrm{g} 0}=\mathrm{S}_{0}$

Cependant la transformation (4) permet de modéliser le processus de génération de houle due à l'action du vent sur le courant et sur l'eau statique.

En effet des expériences précises et à grande échelle, effectuées en aéro-hydrocanal [10] ont montré que la fréquence du maximum du spectre de houle due à l'action du vent $\mathrm{f}_{0 \mathrm{~m}}$ et la hauteur significative de houle $\mathrm{H}_{\mathrm{s}}$, dans le tronçon de développement, peuvent être formulées par les approximations suivantes :

$$
\begin{aligned}
& \frac{u_{*} f_{o m}}{g}=0,939\left(\frac{g X_{\text {eff }}}{u_{*}{ }^{2}}\right)^{-0,354} \\
& \frac{g H_{s}}{u_{*}{ }^{2}}=0,0222\left(\frac{g X_{\text {eff }}}{u_{*}^{2}}\right)^{0,669}
\end{aligned}
$$

où $\mathrm{U} *$ - vitesse de frottement, $\mathrm{X}_{\mathrm{eff}}$ se détermine par la relation (2).

Les relations (7) et (8) sont satisfaisantes pour le vent, les courants contraire et favorable et aussi pour les conditions d'eau statique. Ce résultat confirme, en même temps dans les limites de l'exactitude des expériences, la légitimité de la supposition $S^{\prime}=S_{0}$ prise plus haut sans démonstration.

Les relations (7) et (8) correspondent aux conditions d'eau profonde. Pour la profondeur finie d, en utilisant la condition de développement limite de la houle [11], on peut, au lieu de (8), obtenir la relation de calcul suivante:

$$
\frac{g \bar{H}}{W_{10}{ }^{2}}=0,0017\left(\frac{g X_{\text {eff }}}{W_{10}^{2}}\right)^{0,669} \operatorname{th}\left[84,7 \frac{\left(\frac{g d}{W^{2}}\right)^{0,945}}{\left(\frac{g X_{\text {eff }}}{W_{10}^{2}}\right)^{0,669}}\right]
$$

où est accompli le passage à l'amplitude moyenne $\bar{H}$ et à la vitesse théorique du vent mesurée à une hauteur de $10 \mathrm{~m}$ au dessus du niveau de la mer $\mathrm{W}_{10}$. 
Dans le cas de canal ou d'estuaire étroit apparaît un facteur supplémentaire d'influence sur la génération de la houle; il s’agit de la largeur du canal. La résolution correspondante s'effectue dans ce cas en introduisant la longueur effective de fetch qui se détermine comme suit:

$$
\frac{\phi}{x}=\frac{\int_{0}^{\theta^{\prime}} p(\theta) x^{\prime}(\theta) d \theta}{\int_{0}^{\theta^{\prime}} p(\theta) x d \theta}
$$

où : $p(\theta)$ - fonction pondérale déterminant l'efficacité d'agitation par le vent agissant sous un angle d'incidence $\theta$ par rapport à l'horizontale, $x^{\prime}$ - la longueur de fetch pour ce cas, $\theta^{\prime}$ grandeur du secteur dans lequel le vent est efficace. En particulier, pour $\theta^{\prime}=\frac{\pi}{6},: p(\theta)=1$ on obtient :

$\frac{\phi}{\mathrm{x}}=\frac{6}{\pi}\left[\ln \operatorname{tg}\left(\frac{1}{2} \operatorname{arctg} \frac{\mathrm{B}}{2 \mathrm{x}}+\frac{\pi}{4}\right)-1,317 \frac{\mathrm{B}}{2 \mathrm{x}}-\frac{\mathrm{B}}{2 \mathrm{x}} \ln \operatorname{tg}\left(\frac{1}{2} \operatorname{arctg} \frac{\mathrm{B}}{2 \mathrm{x}}\right)\right]$

où B- largeur du canal.

En présence d'écoulement dans un canal étroit ou dans un estuaire, le courant liquide et la largeur limitée du canal influent simultanément sur la génération de houle due à l'action du vent. La vérification de la méthode de prévision de la houle dans les grands canaux, effectuée par les auteurs sur la base de l'utilisation de la notion de la longueur effective (2) et (10), a montré que cette méthode s'accorde bien avec les résultats de mesure.

En s'approchant du rivage, la houle commence à interagir avec le fond et l'écoulement dans la zone d'influence de la veine liquide de l'ouvrage de rejet ou de prise d'eau. En conséquence de cette interaction se produisent une transformation de la houle et une variation des caractéristiques cinématiques du courant liquide. Examinons certains problèmes avec le cas d'approche frontale de la houle sur un jet symétrique, ce qui permet de se limiter dans l'analyse à un problème unidimensionnel.

Le premier problème à résoudre dans ce cas demeure le calcul du champ de vitesses dans le jet en l'absence de l'écoulement. Pour un tel calcul on peut utiliser certaines relations connues, par exemple [12] pour le cas de rejet de veine liquide par une marche on a:

$\mathrm{u}_{\mathrm{mx}}=\frac{\text { const }}{\sqrt{\mathrm{x}}}$

où $\mathrm{x}$ - distance du point de rejet, $\mathrm{u}_{\mathrm{mx}}$ - vitesse moyenne sur l'axe de la veine. Dans le cas de raccordement de l'ouvrage de rejet au bassin de restitution par un talus de pente $1 / \mathrm{m}$ il est plus commode d'utiliser la relation suivante [13] :

$\frac{u_{m x}}{u_{0}}=6,4 \frac{h}{x+a}$

où $\mathrm{a}=\mathrm{mh}$, h- tirant d'eau du courant de rejet dans la section initiale, $\mathrm{u}_{0^{-}}$vitesse à la sortie du jet. Pour le calcul du champ de vitesses du jet dans des cas difficiles on utilise des méthodes numériques permettant la prise en considération totale des conditions aux frontières [14]. 
Le courant de rencontre à gradient longitudinal négatif de vitesse, de même que la profondeur régressive près du rivage, entraînent l'accroissement de l'amplitude et la diminution de la longueur d'onde. Cet effet peut bien être décrit par les résolutions suivantes en approximation adiabatique [6]:

$$
\begin{aligned}
& \sqrt{\frac{d / L_{0}}{L / L_{0}} \operatorname{th}\left(2 \pi \frac{d / L_{0}}{L / L_{0}}\right)}=\sqrt{\frac{d}{L_{0}}}\left[1-\frac{u d / L_{0} C_{0}}{\left(\frac{L}{L_{0}} \cdot \frac{d}{L_{0}}\right)}\right] \\
& \frac{H}{H_{0}}=\left[\frac{1}{L / L_{0}} \operatorname{th}\left(2 \pi \frac{d / L_{0}}{L / L_{0}}\right)\right]^{1 / 4}\left[\frac{1}{\operatorname{th}\left(2 \pi \frac{d / L_{0}}{L / L_{0}}\right)(1+G)+2 \frac{u d / L_{0} C_{0}}{d / L_{0}}}\right]^{1 / 2}
\end{aligned}
$$

où $\mathrm{L}, \mathrm{H}$ - respectivement longueur d'onde et amplitude de la houle pour une profondeur $\mathrm{d}$ et une vitesse de courant $\mathrm{u}$ (avec $\mathrm{u}>0$ pour le courant favorable et $\mathrm{u}<0$ pour le courant de rencontre), $\mathrm{L}_{0}, \mathrm{C}_{0}$ et $\mathrm{H}_{0}$ sont respectivement : la longueur d'onde, la célérité de phase et l'amplitude de la houle en eau profonde hors de la zone d'influence du courant, $L_{0}=\frac{g^{2}{ }^{2}}{2 \pi}$, $\mathrm{C}_{0}=\frac{\mathrm{gT}_{\mathrm{a}}}{2 \pi}, \mathrm{G}=\frac{2 \mathrm{kd}}{\mathrm{sh} 2 \mathrm{kd}}$, (où : $\mathrm{T}_{\mathrm{a}}$ - période absolue de la houle et $\mathrm{k}$ - nombre d'onde). Les données expérimentales de différents auteurs [6] satisfont aux relations (14) et (15).

La diminution de la vitesse de groupe et l'accroissement de la cambrure de la houle en propagation sur un courant contraire peut aboutir à l'effet particulier de blocage ou d'arrêt ou encore de déferlement de la houle. L'arrêt de la houle par le courant de rencontre se détermine par la condition cinématique suivante:

$\mathrm{C}_{\mathrm{ga}}=\mathrm{u}+\mathrm{C}_{\mathrm{gr}}=0$

Au voisinage des points correspondants à la condition (16), la résolution adiabatique (15) n'est pas applicable. Pour la détermination approximative de l'amplitude de la houle au voisinage du blocage, il existe des estimations obtenues par l'intégrale d'Airy, par exemple [15]:

$$
\frac{H_{\max }}{H_{0}}=2\left(\frac{\sigma_{a}}{4|\partial u / \partial x|}\right)^{\frac{1}{6}}
$$

où $\mathrm{H}_{\max }-$ amplitude maximale de la houle dans la zone de blocage, $\sigma_{\mathrm{a}}=2 \pi / \mathrm{T}_{\mathrm{a}}$.

Une série d'expériences sur la propagation des ondes gravitiques sur un courant de rencontre horizontal hétérogène a été effectuée par les auteurs. La variation des vitesses d'écoulement a été opérée grâce à des parois supplémentaires verticales convergentes disposées dans un bassin à houle. Les résultats obtenus sont représentés dans les figures $\mathrm{N}^{\circ} 03$ et 04 
Figure3: Amortissement de la vitesse superficielle sur l'axe x de la veine liquide plane

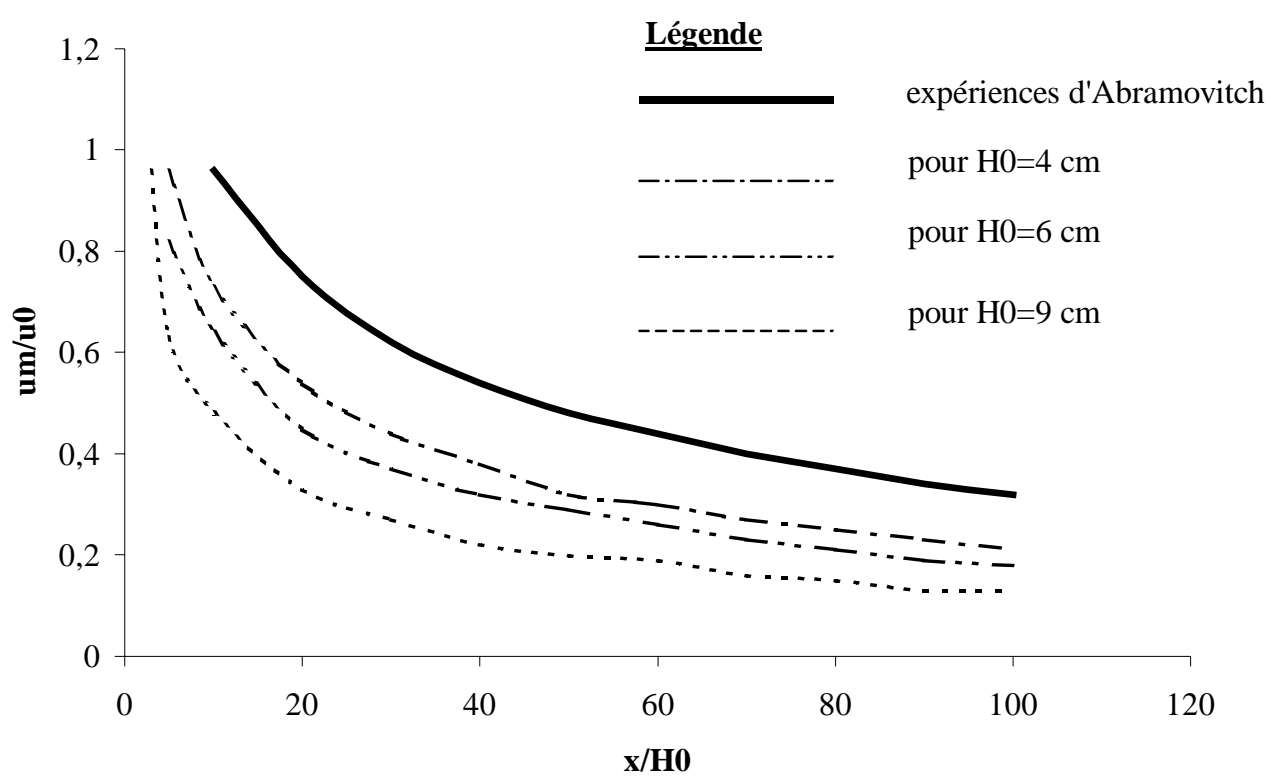

Fig.Nº4: Etalement de la veine liquide sous l'action de la houle (conditions expérimentales: $\mathrm{m}=0$ et $\mathrm{d}=60 \mathrm{~cm})$

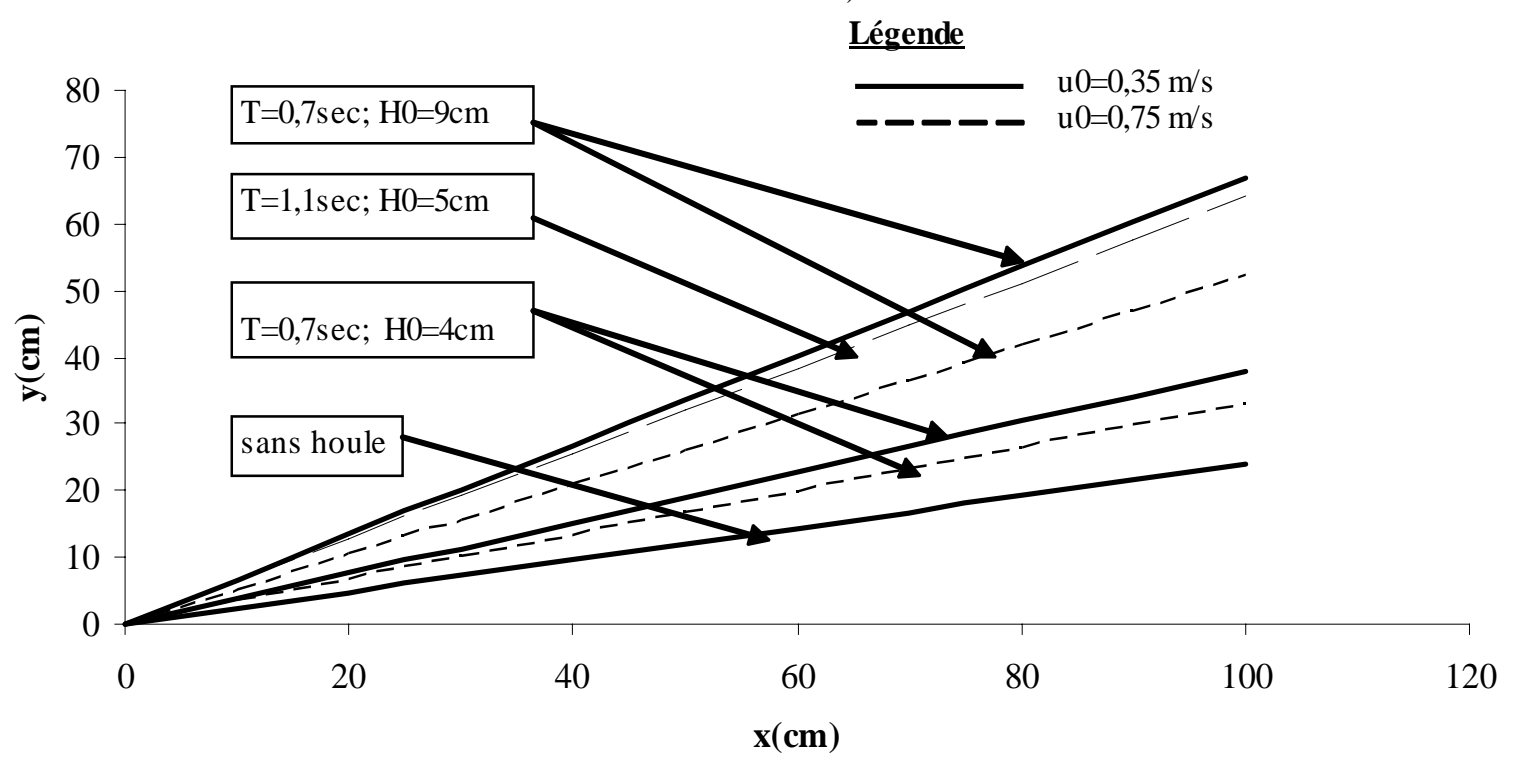

La position des points de blocage de la houle dans ces expériences correspondait bien à la condition (16). Les amplitudes de la houle au voisinage des points de blocage augmentaient de deux fois environ comparativement à l'amplitude initiale. Après la zone de blocage les ondes superficielles disparaissaient pratiquement. Le blocage de la houle était suivi de la destruction des ondes. 
Dans les expériences, les ondes les plus longues déferlaient avant même d'atteindre la condition (16). La question de déferlement de la houle sur les courants de rencontre est actuellement peu élucidée. Pour l'évaluation on utilise dans la plupart des travaux le critère de Miche de cambrure limite de la houle, soit:

$\mathrm{M}_{\mathrm{cr}}=0,142$ thkd.

D’après nos expériences, le critère (18) décrit de façon satisfaisante la position du point de déferlement. Cependant notons qu'il existe des expériences montrant que le déferlement sur le courant de rencontre a lieu pour des cambrures inférieures à $\mathrm{m}_{\mathrm{cr}}$. Il est intéressant de constater que dans les expériences le train d’ondes apparaissant après déferlement de la houle est brusquement bloqué par le courant de rencontre. Ainsi pour les ondes gravitiques réelles considérées dans le calcul des ouvrages hydrotechniques, le blocage et le déferlement de la houle par le courant de rencontre sont étroitement liés.

L'influence inverse de la houle sur le courant de rencontre aboutit à l'accroissement de l'angle d'étalement en plan de la veine liquide et à la diminution des vitesses longitudinales. Ces effets ont été confirmés par des expériences que nous avons effectuées dans un bassin où étaient produits des courants et des lames debout.

Concernant les problèmes d'affouillement local et général, la question de détermination, avant tout, des vitesses de fonds dans les conditions de propagation de lames favorables et de lames debout apparaît nécessaire. Pour résoudre ce problème il existe actuellement une seule méthode fondée [6], dans laquelle les vitesses de courant s’ajoutent aux célérités d'onde en considérant la transformation de la houle sous l'action du courant. Ainsi pour la houle on applique la théorie de Stokes de troisième ordre. Comme l’ont montré les expériences, l'approche la plus commode est prise pour la condition:

$\frac{\mathrm{u}}{\mathrm{u}^{\prime}}>1$

où u- vitesse du courant, u'- vitesse ondulatoire horizontale maximale pour l'horizon z [16].

Le calcul du cône d'affouillement aval par le courant peut s'effectuer par les méthodes connues. Par exemple la méthode itérative de comparaison des vitesses de fond à profondeur croissante avec les vitesses admissibles relatives au matériau donné. Dans le cas de formation de fosse d'affouillement, les matériaux enlevés sont transportés et déposés plus au large constituant ainsi une barre marine. La transformation de la houle dans ce secteur doit être déterminée en tenant compte de la barre marine, de la fosse d'affouillement du courant et des effets d'exhaussement du plan d'eau.

L'expérience de calcul montre qu'en ce qui concerne les ouvrages maritimes de rejet pour lesquels la houle de projet est celle de fréquence 1\% (une fois sur 100 ans) et de longueur d'onde de l'ordre de $150 \mathrm{~m}$ en eau profonde, la barre marine ainsi que la fosse d'affouillement peuvent ne pas provoquer de réfraction ni de dissipation considérable des ondes. La position du lieu de déferlement de la houle de projet, en tenant compte des particularités de la configuration du fond et des courants, détermine les grandeurs des charges dues à l'action des vagues sur les ouvrages maritimes. Notons que le problème ici est essentiellement spectral, c'est pourquoi, tant dans les calculs que dans la similitude hydraulique, il faut en tenir compte. Du point de vue pratique, on peut conclure que les plus petites ondes peuvent donner des grandes charges et provoquer des déformations néfastes du fond comparativement à l'action des plus grandes ondes. Cependant, dans la similitude hydraulique et dans les calculs, il faut prendre en considération le caractère spectral de la houle, quitte à répéter les expériences et calculs pour chaque onde harmonique. 
L'application de la houle sur le courant peut entraîner des variations des grandeurs (et de la direction dans le cas de lame debout) du flux total des sédiments. Nous avons élaboré un modèle de transport de sédiments mixtes par la houle et le courant de rencontre [17] et effectué un grand cycle d'expériences pour la mesure du débit solide pour différents rapports d’onde, de courant et de caractéristiques des sédiments. Les résultats de calcul par la méthode étudiée s'accordent de façon satisfaisante avec nos propres données expérimentales ainsi qu'avec celles des expériences analogues effectuées par d'autres auteurs.

Pour le débit spécifique des sédiments transportés en charriage et en suspension on a obtenu la relation suivante:

$$
\begin{aligned}
& \frac{\mathrm{q}_{\mathrm{s}}}{\mathrm{q}_{\mathrm{so}}}=\frac{9}{16} \pi^{4}\left(\frac{\mathrm{H}_{\mathrm{p}}}{\mathrm{u} \mathrm{T}_{\mathrm{a}}} \mp \frac{\mathrm{H}_{\mathrm{p}}}{\overline{\mathrm{L}}}\right)\left(\frac{\mathrm{H}_{\mathrm{p}}}{\overline{\mathrm{L}}}\right) \operatorname{sh}^{-6} \frac{2 \pi \mathrm{d}}{\overline{\mathrm{L}}}+ \\
& +\frac{3}{2} \frac{\mathrm{u}_{\mathrm{s}}}{\mathrm{u}} \pi^{2}\left(\frac{\mathrm{H}_{\mathrm{p}}}{\mathrm{uT}_{\mathrm{a}}} \mp \frac{\mathrm{H}_{\mathrm{p}}}{\overline{\mathrm{L}}}\right)^{2} \operatorname{sh}^{-2} \frac{2 \pi \mathrm{d}}{\overline{\mathrm{L}}}\left[1+\frac{9}{16} \pi^{2}\left(\frac{\mathrm{H}_{\mathrm{p}}}{\overline{\bar{L}}}\right)^{2} \operatorname{sh}^{-6} \frac{2 \pi \mathrm{d}}{\overline{\mathrm{L}}}\right]+\left(\frac{\mathrm{u}_{\mathrm{s}}}{\mathrm{u}}\right)^{3}
\end{aligned}
$$

où $\mathrm{q}_{\mathrm{so}^{-}}$débit des sédiments transportés par le courant uniquement, $\mathrm{H}_{\mathrm{p}}$ - amplitude de projet de houle de $30 \%$ de fréquence pour le transport solide, $\bar{L}$ - longueur d'onde moyenne, $\mathrm{u}_{\mathrm{s}}$ - vitesse stationnaire de fond pour l'action combinée de la houle et du courant, déterminée par la relation suivante:

$$
\frac{\mathrm{u}_{\mathrm{s}}}{\mathrm{u}}=\quad \begin{aligned}
& \text { 1,(pour le courant favorable) } \\
& 2,5 \pi^{2}\left(\frac{\mathrm{H}_{\mathrm{p}}}{\mathrm{HT}_{\mathrm{a}}} \overline{\frac{H_{p}}{\bar{L}}}\right)\left(\frac{\mathrm{H}_{\mathrm{p}}}{\overline{\mathrm{L}}}\right) \operatorname{sh}^{-2} \frac{2 \pi \mathrm{d}}{\overline{\mathrm{L}}}-1, \text { (pour le courant de rencontre, avant } \\
& \text { déferlement) } \\
& 1, \text { (pour le courant de rencontre, après déferlement) }
\end{aligned}
$$

Dans les relations (20) et (21) le signe (-) correspond au courant favorable tandis que le signe $(+)$ correspond au courant de rencontre. La dépendance (20) peut être utilisée pour la détermination de l'influence de la houle sur le transport solide et sur le caractère de déformation du fond dans la zone de l'interaction de la houle et des courants de rejet ou de prise d'eau en mer.

Comme il l'a été souligné, l'étude des ouvrages de rejet et de prise d'eau en mer nécessite également une bonne connaissance de la dynamique des sédiments dans la zone de rivage contigu. Cette dynamique est aussi influencée par les courants littoraux provocants le déplacement des sédiments et une déformation du rivage. Pour le transport littoral des sédiments il existe des relations empiriques vérifiées par des données naturelles [9].

\section{Conclusion}

L'interaction de la houle et des courants dans la zone de raccordement des ouvrages de rejet et de prise d'eau avec le plan d'eau agité influe considérablement sur la cinématique des ondes. Dans cette zone on observe la transformation des paramètres de la houle et la perturbation du transit littoral, conséquence inévitable d'une érosion du rivage. Cependant les résultats de modélisation du processus de génération de la houle sur les courants permettent d'élaborer une méthodologie de calcul hydraulique des ouvrages de rejet et de prise d'eau en mer. 


\section{Références}

1.OFITSEROV A.S. - Problèmes hydrauliques de prise d'eau (en russe), Gosstroiïzdat, Moscou, 1952

2.OFITSEROV A.S. - Prises d'eau maritimes en eau peu profonde et méthodes de diminution de leur envasement (en russe),Tr. Vodgeo, vip.69, Moscou, izd-vo Vodgeo, 1977

3.SMIRNOV A..A.- Résultats importants de recherche sur l'influence des courants de rive sur les prises d'eau de barrage (en russe),Tr. Conférences de coordination sur l’hydrotechnique, vip. 39, Leningrad,1968.

4.MIKHAILOV K.A. - Ouvrages de prise d'eau pour alimentation en eau potable à partir de sources superficielles (en russe), Moscou, stroiïzdat, 1976.

5.MASS E.I., KANTARJI I.G.- La houle sur le courant stationnaire à profondeur finie (en russe), vodny ressourcy, $\mathrm{N}^{\circ} 1,1978$.

6.KANTARJI I.G. et autres- Hydraulique de la houle due au vent dans les canaux (en russe). Sous rédaction de MASS E.I., izd-vo T.G.OU., Tbilicy, 1984.

7.HASSANE M.- Prévision des éléments de la houle et des affouillements du fond dans la zone de rejet en mer (en russe). Thèse de doctorat Phd- Moscou 1987.

8. «charges et action exercées sur les ouvrages hydrotechniques (par la houle, les glaces et les navires) » (en russe).SniP 2.06.04-82, Moscou, stroiïzdat, 1983.

9. «shore protection manual», U.S. Army Coast.End. Res.Cent., vol.1,1973.

10.KATO H.,TSURUYA H.- Experimental study of wind waves generated on currents, proc.16 ${ }^{\text {th }}$ Coast.Eng.Conf., vol.1, 1979.

11.KRILOV Y.M., STREKALOV S.S., TSIPLOUKHINE V.F.- La houle de vent et son action sur les ouvrages(en russe). Leningrad, guidrometeoïzdat, 1976.

12.ABRAMOVITCH G.N.- The theory of turbulent jets, M.I.T. Press Cambridge,Mass. 1963.

13.KHINTSE I.O.- La turbulence, son mécanisme et sa théorie (en russe). Moscou, fizmatguiz, 1963.

14.BENGUE J.P., HANGUEL A.,VIOLLET P.L.- Engineering applications of computational hydraulics, Pitman, Landon, 1982.

15.POKAZEEV K.V., ROZENBERG A.D.,- Étude en laboratoire des ondes gravitiques capillaires régulières sur les courants (en russe). Okeanologuia, vip.4, 1983.

16.KNOLL D.A., HERBICH J.B.,- Simultaneans wave and current forces on a pipline. Proc. $17^{\text {th }}$ Coast. Eng. Conf., vol.2, 1981.

17.HASSANE M., MASS E.I., KANTARJI I.G,.., Transport solide par la houle et les courants dans les grands canaux. Revue construire $N^{\circ} 19$, Alger, 1987

18.DREIZIS Y.I., KANTARJI I.G., PELINOVSKY E.I.,- Filtration des ondes par les courants de déplacement (en russe). Vodny ressourcy, N¹, 1986.

19.KANTARJI I.G., KOSTIAN V.O.,TSIVTSIVADZE N.C.,- Modèle mathématique de l'évolution de plage entre épis (en russe). Recueil des travaux de TSNIIS, Moscou, TSNIIS, 1984.

20.P.F. DEMENT et B.QUETIN- Méthodes et outils utilisés pour l'étude des rejets en mer- application à différents cas d'études- Houille blanche $N^{\circ} 3$ / 4-1990

21.P. Bonneton, V. Marieu, H. Dupuis, N. Sénéchal and B. Castelle, 2004. Wave transformation and Energy Dissipation in the Surf Zone: Comparison Between a Non-linear Model and Field Data. Submitted ICS 2004, Brazil, SI 39 of Journal of Coastal Research 